\title{
Duopolio, diferenciación y escala: un estudio de las estructuras de costos de las administradoras de fondos de pensiones en Bolivia
}

\section{Duopoly, Differentiation and Scale:A study of the cost structures of the Pension Fund Administrators in Bolivia}

Daniel Maceira*

Pablo Adrián Garlati Bertoldi* *

\section{Resumen $^{* * *}$}

Desde los años 80 existen sistemas de pensiones de capitalización individual en América Latina. El marco regulatorio debió perfeccionar sus instrumentos de intervención, a fin de reducir potenciales fallas de mercado. Una herramienta clave en este proceso es identificar los niveles de concentración del mercado y las estructuras de costos de las firmas, a fin de determinar si las administradoras de fondos de pensiones (AFPs) absorben rentas monopólicas o si se caracterizan por funciones de costos decrecientes. Utilizando datos del

* Ph.D. en Economía. Investigador Titular del Centro de Estudios de Estado y Sociedad (CEDES), Buenos Aires, Argentina. Contacto: danielmaceira@cedes.org

** Master en Economía. Contacto: adriangarlati@gmail.com.

***El presente trabajo resume algunas de las conclusiones alcanzadas para el caso boliviano de una investigación elaborada con el apoyo de la Asociación Internacional de Organismos de Supervisión de Fondos de Pensiones (AIOS), y contó con financiamiento provisto por el Banco Mundial. Se agradece a las autoridades de la AIOS por la información suministrada y los comentarios aportados. Especial reconocimiento a Mercedes Kweitel, quien brindó su apoyo en la sección econométrica, y a Georgina Gahr, asistente de investigación del CEDES, por su colaboración a lo largo de este proyecto. Las conclusiones son de entera responsabilidad de los autores, y no necesariamente reflejan aquellas de las instituciones patrocinantes. 
sistema privado de pensiones de Bolivia para el periodo 1996-2004, se estimaron en forma paramétrica y semiparamétrica las funciones de costos de las AFPs. Se encontró una relación significativa y negativa entre costos medios y número de afiliados, y un bajo efecto de las tasas de rendimiento de los fondos sobre los costos medios del sistema. Complementariamente, las normas regulatorias no parecen estar operando directamente sobre los costos medios, sino a través del número de afiliados.

Palabras clave: sistema de pensiones, mercado, normas regulatorias, Bolivia

\begin{abstract}
Private pension systems exist in Latin America since the 80 's. The regulatory framework improved its instruments of intervention specially focused on enhacing competitiveness and reducing potential market failures. A key tool is to identify market concentration and cost behaviors is to determine if Pension Funds Administrators (AFPs) are absorbing monopolic rents or if they are characterized by decreasing costs. Using data of the private pension system in Bolivia during the period 1996-2004, we estimated the AFPs cost functions using parametric and semi-parametric methods. A significative and negative relationship was founded between average costs and the number of affiliates; and a small effect of funds profit rates on the systems ' average costs. Also, regulations do not seem to be affecting firms' costs directly but through the number of affiliates.
\end{abstract}

Keywords: pension system, market, regulation, Bolivia

Clasificación/Classification JEL: C14, C23, D24, L1 1, L52

\title{
1. Introducción
}

Los años 80 iniciaron en América Latina una serie de reformas estructurales, donde avanzó la participación del sector privado en la oferta de bienes y servicios que se encontraban en manos del Estado, siendo el sistema de pensiones uno de ellos. Ello ha implicado un desafío regulatorio, especialmente en la prestación de servicios sociales.

De acuerdo a los impulsores de estas reformas, la caída sistemática de la tasa de fertilidad y el aumento de la esperanza de vida dificultaban la sustentabilidad de los mecanismos públicos 
de "pay-as-you-go", dando lugar al nacimiento de fondos privados de gestión. A partir de entonces se ha debatido sobre las ventajas y desventajas de cada alternativa.

El debate incorpora aspectos vinculados con la inequidad en la provisión de servicios de pensiones, con altos costos y cargas administrativas, como plantea Trigg y Lowe (2011) a partir de comparaciones internacionales y una especial aplicación para el caso inglés. Asimismo, Ferreiro y Serrano (2011) sugieren que las capacidades de respuesta de cada modelo surgen de los modos de acceso a la información y la gestión de fondos de los modelos existentes, dependiendo de experiencias particulares la definición de sus capacidades de respuesta, como también de su estructura normativa y los resguardos institucionales en los cuales se apoyan (como plantea Larraín Villanueva (2012) para el caso chileno).

El trabajo de Barr y Diamond (2010) resume el debate sobre las reformas de los sistemas de pensiones, aportando un marco teórico para el análisis. Indica que cada caso nacional se enfrenta a diferentes riesgos, y por tanto las respuestas no necesariamente son uniformes, por lo que no existe un modelo ideal. En todo caso, la información al usuario, de modo de maximizar su capacidad de consumo inter-temporal, resulta el eje de análisis, permitiendo reducir el riesgo de pobreza en los últimos tramos del ciclo vital.

Desde otra perspectiva, Rofman y Oliveri (2011) provee una revisión exhaustiva de los sistemas de pensiones latinoamericanos, identificando las particularidades de cada caso.

Dentro de este marco, las reformas de los sistemas de pensiones mediante regímenes de capitalización individual han permitido que la recaudación y gerencia de fondos se realice por medio de sociedades comerciales (Administradoras de Fondos de Pensiones, AFPs en Bolivia), operando en un contexto de mercado administrado.

El supuesto de competencia bajo un esquema de producto homogéneo (pensiones), dio lugar a la compulsa, no solo por precios (estructura de comisiones), sino también mediante mecanismos de diferenciación asociados a percepciones de calidad, confiabilidad, reputación y rentabilidad.

El marco regulatorio debió perfeccionar sus instrumentos de intervención, a fin de reducir fallas de mercado asociadas con información asimétrica e imperfecta. Tales instrumentos incluyeron cambios en los modos de fijar comisiones y niveles de costos administrativos, como también en los criterios para la confección de portafolios de activos. 
Un trabajo elaborado para el caso boliviano (Evia Vizcaria y Fernández Moscoso, 2005) muestra que la reforma previsional en el seguro de largo plazo ha alterado las características de la cobertura del sistema con respecto a la situación previa a la reforma. Usando especificaciones de un modelo probit, encuentra que antigüedad, sexo y duración de la jornada laboral determinan la afiliación al esquema jubilatorio del país, en tanto que la educación y la edad continúan siendo elementos significativos en la demanda de estos servicios.

Desde una perspectiva de costos de operación, y particularmente orientado al objetivo principal de este trabajo, el análisis de los sistemas de capitalización individual de pensiones muestra la necesidad de establecer niveles de concentración consistentes con una industria de producción de servicios (Apella y Maceira, 2004). El presente trabajo propone modelar y estimar funciones de costos en el mercado boliviano de AFPs, contando para ello con información provista por la Asociación Internacional de Organismos de Supervisión de Fondos de Pensiones (AIOS) para el periodo 1996-2008.

En las dos secciones siguientes se presenta, respectivamente, el marco teórico que promueve este estudio y una revisión de los antecedentes directos de este trabajo.

La cuarta sección describe caracteristicas salientes del mercado de AFP, para luego avanzar en la descripción de variables y la implementación econométrica en las secciones quinta y sexta, en la estimación tanto paramétrica como semiparamétrica de los modelos presentados en las secciones anteriores. Ello permite una comparación amplia y sistemática, que aporta al conocimiento sobre el comportamiento de la función de costos en este mercado bajo modos alternativos de medición.

Finalmente, la quinta sección propone un debate analítico y de política pública a la luz de los hallazgos presentados a lo largo de la investigación.

\section{Marco teórico}

Como fuera planteado en otros trabajos (Apella y Maceira, 2004), "el número de firmas participantes en el mercado o la tasa de concentración del mismo, no definen por sí solos su carácter perfectamente competitivo u oligopólico". Un mercado "perfectamente competitivo" implica que cada firma participante no tenga influencia sobre las señales del mercado y por tal motivo se comporte como tomador de precios. Este hecho encuentra fundamento en 
la homogeneidad del bien o servicio ofrecido, como también en los niveles de eficiencia alcanzados por la industria.

La falta de homogeneidad en el servicio prestado por el mercado de las AFPs puede basarse en mecanismos de diferenciación reales o aparentes, que hacen que cada firma, al decir de Chamberlin (1956), operen en una suerte de submercado, enfrentando funciones de demanda no horizontales y permitiendo influir estratégicamente en la operatoria del sistema.

Estas estrategias de diferenciación, más allá de afectar las estructuras de costos de las empresas proveedoras de servicios, establecen la necesidad de analizar cuáles son los mecanismos a partir de los cuales las AFP compiten. La evidencia recogida en la mayoría de los mercados surgidos con los nuevos sistemas de pensiones muestra una muy baja elasticidad precio de la demanda junto con altos costos de promoción y comercialización. Así, la competencia no está dada a nivel de precios únicamente, sino a través de una fuerza de ventas que captura a la demanda en función de otras variables (CEF, 2008).

Sin embargo, el aumento en la tasa de concentración en una industria de servicios no necesariamente se vincula con la presencia de ganancias monopólicas, sino con la posibilidad de operar en tramos más eficientes de sus funciones de producción y costos. En tales casos, actuar sobre la estructura del mercado (en este caso el número de firmas) no se corresponde con una reacción regulatoria necesariamente adecuada, en tanto limitaría la posibilidad de obtener rentas ricardianas, identificadas con la presencia de economías de escala.

De probarse esa hipótesis, el ente regulador debería concentrarse en identificar la estructura real de costos del sector y facilitar que las rentas de eficiencia no beneficien solamente al productor de servicios, sino que derramen sobre los precios abonados por el consumidor. Desde esta perspectiva, aproximarse a la "verdadera" estructura de costos de la industria de pensiones favorece el accionar de la autoridad de control, en tanto permite diferenciar entre situaciones posibles, maximizando el poder de intervención ${ }^{1}$.

En tanto el conocimiento de la verdadera estructura de costos no es posible de acceder en un marco de información asimétrica en contra del ente regulador, establecer un método econométrico de estimación constituye una herramienta de política pública. En la misma

1 El diseño regulatorio óptimo aplicable a un sistema o régimen previsional en el que participan prestadores privados requiere elaborar las reglas básicas para que el equilibrio de mercado resultante se aproxime lo más posible al óptimo social. En tanto que los agentes interactúan y compiten basados en dichas reglas, el planteo requiere conocer en detalle cómo reaccionan éstos ante las mismas para evitar efectos indeseados e incorporar dichas respuestas en el ejercicio de diseño óptimo (Auguste y Urbiztondo, 2008) 
dirección, poder identificar el efecto de la normativa regulatoria sobre las estructuras de costos de la oferta de pensiones privadas contribuye a diferenciar las intervenciones acertadas, aumentando la información sobre las herramientas más adecuadas de impacto (Apella y Maceira, 2005).

En base a este enfoque, se propone estimar estructuras de costos medios para las AFP participantes del mercado de pensiones de Bolivia, identificar y testear la incidencia de hitos regulatorios que operaron en el mercado y debatir sobre el impacto de tales hallazgos sobre la acción regulatoria del organismo de control.

\section{Metodología y modelo a estimar}

Una vasta literatura antecede y permite establecer argumentos debatidos en trabajos previos en otros países. Galarza y Olivera (2001), y Berdejo, Galarza y Nagamine (2006) plantean la existencia de fuertes barreras a la entrada en el mercado de pensiones privadas de Perú. En particular, concluyen que el elevado nivel de costos hundidos necesarios en la etapa inicial de incursión en el mercado generó una significativa falta de competencia entre las AFPs.

García y Rodríguez (2003), Zepeda y Roldán (2005) y Aguilera (2004) entre otros, estudian, para el mercado privado de pensiones de México, el comportamiento de sus estructuras de costos, encontrando rendimientos crecientes en tecnología. El primero emplea una forma funcional utilizada en la literatura: la especificación cuadrática (Chisari et al., 1998). En tal estudio, los autores construyen una regresión en la que el costo total es explicado por su componente fijo, el número de afiliados, la cantidad de traspasos y la tasa de rendimiento.

Zepeda y Roldán (2005) evidencian que, cuanto menor sea la escala de producción, mayor es la eficiencia en términos de costos. Por su parte, y para el caso de Argentina, Apella y Maceira (2004) y Auguste y Urbiztondo (2004) estiman la hipótesis de economías de escala en la industria de AFPs, con resultados equivalentes.

Finalmente, $C E F$ (2008) incluye lanálisis comparativo delos mercados deadministración de fondos de pensiones en Chile, México, Perú y Argentina (en este último caso antes de la estatización), buscando testear la presencia de economías de escala. El estudio propone un primer intento de ofrecer un análisis entre estos países hasta 2006, introduciendo la discusión comparada con sistemas de pensiones más avanzados. 
La posibilidad de identificar la existencia de economías de alcance y escala ha sido usualmente aproximada por la literatura a través de especificaciones paramétricas. Esto, sin embargo, propone cierta rigidez en el comportamiento de las variables (Cobb-Douglas, Translog, Cuadrático, etc.). De tal forma, imponer esta forma funcional puede llevar a un sesgo de especificación.

La estimación semi-paramétrica propuesta permite, a diferencia del tratamiento tradicional, incluir mayor flexibilidad en las especificaciones de las relaciones planteadas en la literatura, siendo que algunas características de la industria (como economías de escala y alcance) serán planteadas como hipótesis a testear, y no impuestas como parte de las funciones objetivo.

Especificaciones rígidas, como la función translog o diversos modelos que resultan de transformaciones de Box y Cox (1964), son generalmente criticadas por ser aproximaciones polinómicas locales de la verdadera y desconocida función objetivo. En tal sentido, al extrapolar dicha aproximación local a datos globales, el comportamiento de las funciones mencionadas puede diferir significativamente cuando tal comportamiento global se aleje del local (Aguilera y Velázquez, 2005).

En tal sentido, el trabajo utilizará la especificación de una regresión semiparamétrica Kernel en la función de costos basada en Robinson (1988). Asimismo, y como forma aproximada de la relación real entre los costos y las variables-producto a ser consideradas (afiliados y monto de fondos), se realizará una estimación puramente no paramétrica de la función de costos basada en el enfoque de estimación de esperanzas condicionales de Naradaya-Watson².

Un caso particular de las especificaciones puramente no paramétricas es el modelo de regresión parcialmente lineal (MRPL). De acuerdo a ello, los costos medios de la empresa $i$ en el período $t$ pueden expresarse como:

$$
C M e_{i t}=\beta X_{i t}+g(\text { Afiliados })+\eta D+u_{i t}
$$

2 Si bien el Kernel y el ancho de banda (i.e., ventana) considerados remiten a una discusión usual de la literatura, el trabajo utilizará el Kernel de Epanechnikov y una ventana de 0.5 . 
donde

$C M e=\frac{\text { Costos operativos }}{\text { Afiliados }}$

$X_{i t}=$ variables de precios de factores y variables ambientales

$g$ = es la función de costos desconocida que se desea estimar en forma no paramétrica

$D=$ variables dummy que indican cambios regulatorios en el sistema de pensiones.

Afiliados = suma de afiliados registrados más trabajadores asignados por no elección voluntaria del trabajador.

Por su parte, los Costos Operativos ${ }^{3}$ están compuestos por: (i) la remuneración al personal, (ii) los gastos administrativos y comerciales, (iii) la depreciación, (iv) los costos de oportunidad del capital, y (v) el costo del seguro de invalidez y fallecimiento. Particularmente, el costo de oportunidad del capital se obtiene de la ecuación:

$c t \_c o c=$ Patrimonio Neto de AFP $* w_{k}$

Donde $w_{r}$ se estima por medio del modelo CAPM

$$
w_{k}=r_{l}+\beta\left(r_{m}-r_{l}\right)+r p
$$

tal que:

$r-l .=$ tasa de interés libre de riesgo (bono de Estados Unidos a treinta años),

$r-m$ = retorno de una cartera diversificada de acciones (variación del índice S\&P 500),

$r p=$ riesgo país

$\beta$ = índice de correlación simple del retorno de las acciones de la empresa en cuestión y el mercado en su conjunto.

3 Todas las variables monetarias se pasaron a pesos constantes usando el Índice de Precios al Consumidor (IPC) publicado por Banco de México. Se consideran las AFOREs posteriores a las fusiones para permitir el análisis temporal (se usaron variables dummies por cada fusión porque facilita identificar el peso de las mismas en el desarrollo de los costos medios de mercado). 
Por su parte, las variables de precios de factores están definidas como:

r, que se estima por al modelo CAPM (w_r)

$$
w_{l}=\frac{\text { Gasto en Personal de Venta }}{\text { Cantidad de promotores }}\left(\mathrm{w}_{-} \mathrm{l}\right)
$$

Finalmente, las variables ambientales son: (i) Rentabilidad de los fondos, (ii) Valor de los fondos, (iii) Cantidad de traspasos positivos, (iv) Logaritmo del tiempo, (v) Cantidad de promotores, y (vi) Cantidad de sucursales.

De la ecuación a estimar surge que Eu $\mid X$, Afiliados, $D=0$, siendo desconocida la forma funcional de $g \cdot$. Bajo las consideraciones realizadas, la estimación se realiza siguiendo a Robinson (1988), siguiendo una secuencia de estimación iniciada con $\beta$, para luego hacer lo propio con $\eta$ y en tercer lugar $g \cdot g(\cdot)$.En la primer etapa se obtiene un estimador consistente de $\beta$ para ambos valores de la variable dummy por separado. En la segunda etapa, por su parte, siguiendo a Chevalier y Ellison (1997), se calcula un estimador consistente de $\eta$ usando $\eta=g^{1}(\cdot)-g^{0}(\cdot)$, donde $g^{1}(\cdot)$ y $g^{0}(\cdot)$ surgen de regresiones kernel de $C m e_{i t}-\beta X$ en

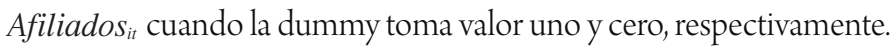

Se planteará en primera instancia una comparación entre especificaciones alternativas de un modelo paramétrico estimado a partir de un panel con efectos fijos a través de AFPs. Posteriormente se compara gráficamente el ajuste del modelo paramétrico y el no paramétrico, estableciendo las diferencias de ajustes entre ambas metodologías.

\section{El mercado de pensiones}

A lo largo del período estudiado (1997-2008), Bolivia presenta un mercado de pensiones reducido, con la participación de dos únicas firmas compitiendo por el liderazgo. Si bien no se ha alterado significativamente en el tiempo la distribución de las participaciones relativas, se observa un leve incremento en la brecha entre ambas, graficada a través de la línea de "rango" en cada una de las figuras presentadas a continuación.

El comportamiento de los fondos muestra un crecimiento del mercado (gráfico 1), un nivel significativo de traspasos entre firmas (gráfico 2) y conductas diferenciales entre firmas entre 2002 y 2005 (gráfico 3). A pesar de ello, la evolución de la rentabilidad es similar, con diferencias mínimas entre ellas (gráfico 4). Estos argumentos, presentados conjuntamente (crecimiento de escala, volatilidad en traspasos, conductas diferenciales en fondos y patrones de 
rentabilidad similares y decrecientes), sugieren la presencia de mecanismos de diferenciación de producto, con potencial impacto sobre las estructuras de costos de las firmas.

Finalmente, el gráfico de costos operativos en el tiempo muestra una tendencia creciente, con poca dispersión entre empresas (gráfico 5).

\section{Gráfico 1: Afiliados}

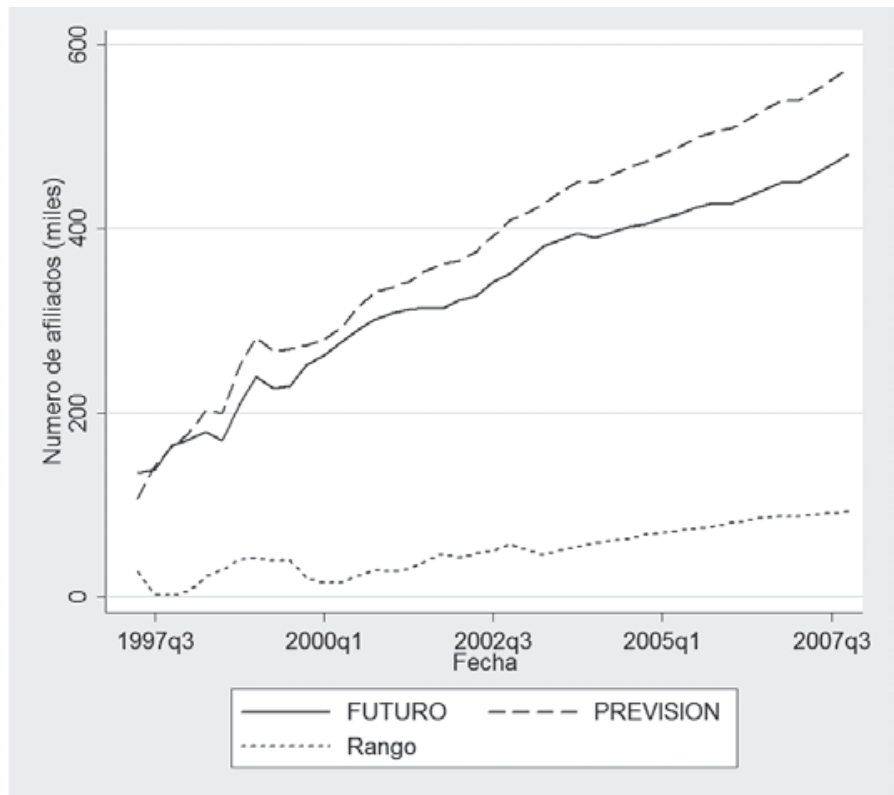

Fuente: Elaboración propia en base a datos de la SPVS 
Gráfico 2: Traspasos

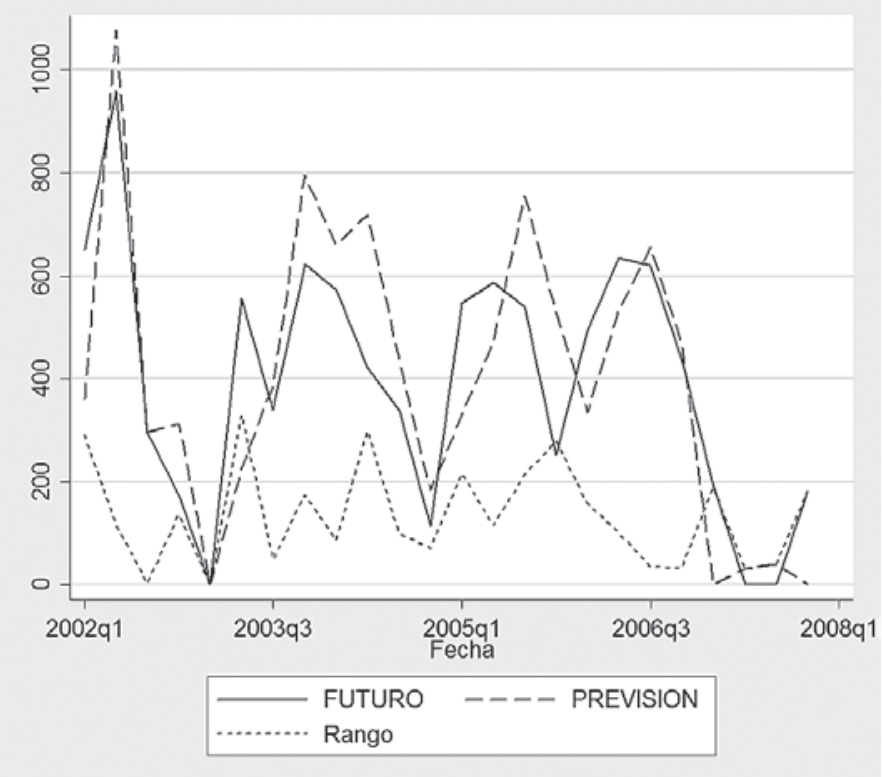

Fuente: Elaboración propia en base a datos de la SPVS 


\section{Gráfico 3: Valor de los Fondos}

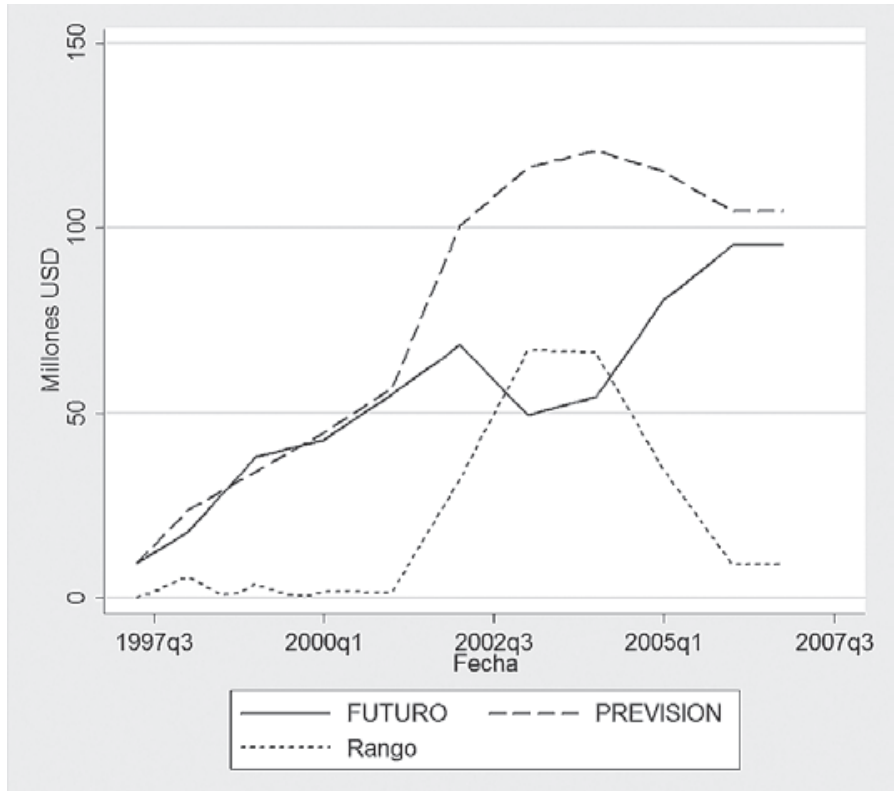

Fuente: Elaboración propia en base a datos de la SPVS 
Gráfico 4: Rentabilidad de los Fondos

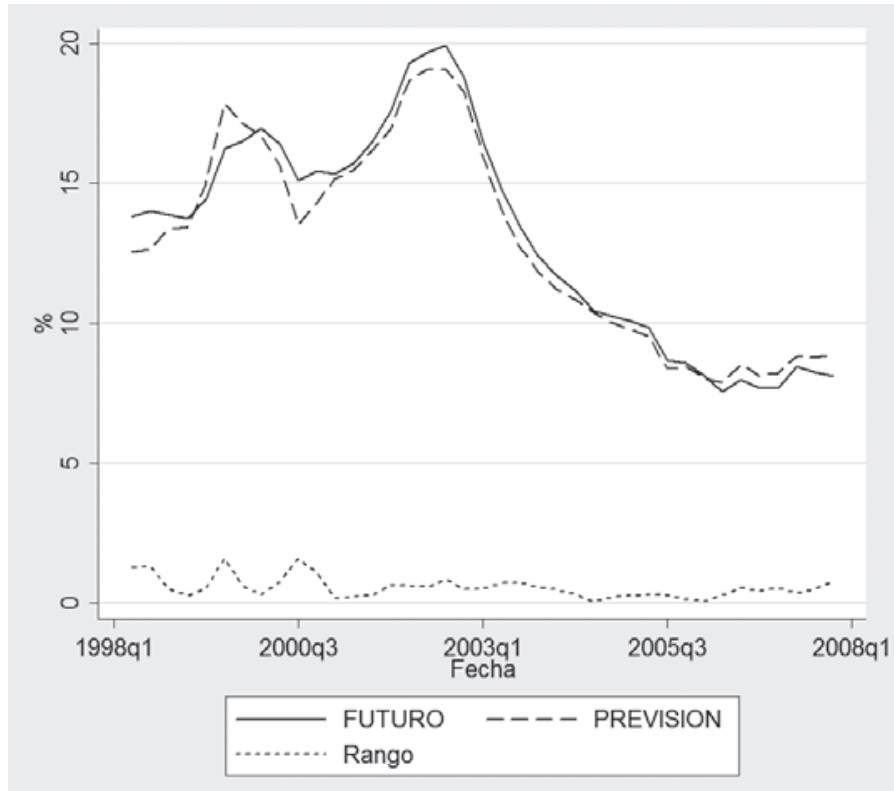

Fuente: Elaboración propia en base a datos de la SPVS 


\section{Gráfico 5: Costos operativos}

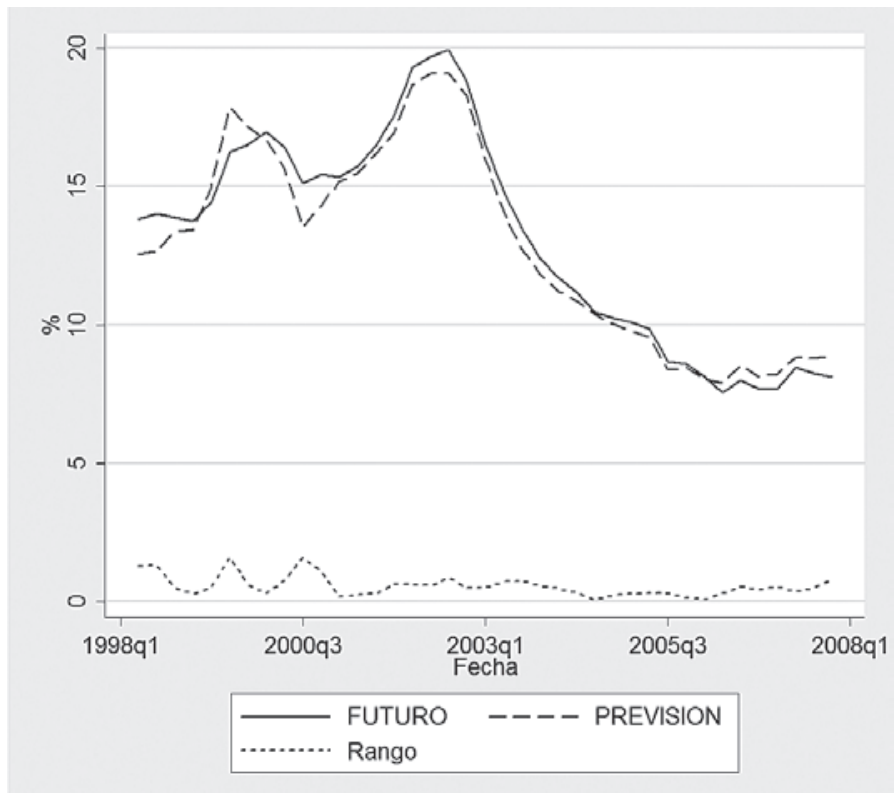

Fuente: Elaboración propia en base a datos de la SPVS

\section{Análisis de estructuras de costos}

\subsection{Descripción de variables utilizadas}

En base a la información recogida, los siguientes cuadros permiten conocer las características de las variables incluidas en la implementación econométrica del modelo. El primero (cuadro 1) identifica los valores medios y extremos de las variables más relevantes utilizadas, mientras que el cuadro 2 presenta un resumen de las regulaciones desplegadas durante el período bajo análisis. En cada caso, se asienta su fecha de aplicación, acompañada por una sigla que define la variable Dummy que fuera utilizada en las regresiones presentadas en la siguiente sección.

Estas siglas dan cuenta del objeto de la norma, siguiendo el planteo presentado en Apella y Maceira (2004). Según esta propuesta, y en base a lo discutido por la literatura tradicional de organización industrial, los mecanismos de intervención del Estado pueden orientarse a 
generar cambios en las condiciones básicas sobre las cuales se establece el sistema, como en la estructura de la misma, promoviendo cambios que afecten los resultados o el desempeño del sector. Una alternativa es regular sobre la conducta de los actores que afecten el comportamiento estratégico de los mismos, tanto oferentes o financiadores como usuarios. A partir de ello es posible identificar solo dos rubros regulatorios en el caso específico de Bolivia para el período estudiado. El primero de ellos opera sobre el mercado, permitiendo el traspaso sin costos entre firmas. La segunda norma actúa específicamente sobre la oferta, regulando la cartera de activos.

\section{Cuadro 1}

Descripción de variables

\begin{tabular}{lrrr}
\hline Variable & \multicolumn{1}{c}{ Media } & \multicolumn{1}{c}{ Min } & \multicolumn{1}{c}{ Max } \\
\hline Cantidad de Afiliados & 356.35 & 107.56 & 574.806 \\
Costos Medios & 35,46 & 24,16 & 71,32 \\
Costos Operativos & 10.900 .000 & 7.671 .000 & 13.600 .000 \\
Rendimiento del Fondo (\%) & 12,99 & 7,57 & 19,92 \\
Traspasos & 223 & 0 & 1.076 \\
Valor del Fondo & 67 & 9 & 121 \\
\hline
\end{tabular}

- Costos originalmente en términos anuales, proyectados en forma trimestral

- Costos en unidades de moneda local constante

- Valor de los fondos de millones en moneda local constante. Año base: 2007.

Fuente: Elaboración propia en base a datos de la SPVS

\section{Cuadro 2}

\section{Estructura regulatoria}

\begin{tabular}{|c|c|c|c|}
\hline Año & Mes & Concepto & Dummy \\
\hline 1996 & Noviembre & Inicio del Sistema & \\
\hline 2002 & Enero & $\begin{array}{l}\text { Traspasos permitidos sin fijación de limite a la } \\
\text { cantidad de traspasos a realizar por un afiliado } \\
\text { durante el año. Dicho movimiento no tiene costos } \\
\text { para el afiliado y se puede realizar por medio de } \\
\text { vendedores.. }\end{array}$ & $\mathrm{ml}$ \\
\hline 2004 & Octubre & $\begin{array}{l}\text { Decreto Supremo 24469. Art. } 211 \text { se prohibe el } \\
\text { uso de Bonos Corporativos Locales en mercado } \\
\text { primero, instrumentos no registrados, ni transables } \\
\text { cn la Bolsa de Valores de Bolivia. }\end{array}$ & 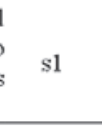 \\
\hline
\end{tabular}

Fuente: Elaboración propia en base a datos de la SPVS 


\section{Implementación econométrica}

\subsection{Introducción}

Elobjetivo deesta sección es presentar, a partirdelos datosobtenidos delaSuperintendencia de Pensiones, Valores y Seguros (SPVS), las estimaciones de costos medios a través de dos metodologías econométricas: paramétricas y semiparamétricas. Se presentarán dos grupos de estimaciones, vinculadas cada una de ellas con las metodologías propuestas, describiendo en primer lugar los resultados alcanzados en los modelos paramétricos, para luego exhibir los coeficientes del componente paramétrico de las estimaciones semiparamétricas. Los resultados finales de las dos especificaciones son comparadas gráficamente. En resumen, los modelos estimados en forma paramétrica fueron

$$
C M e_{i t}=\alpha+\gamma \ln \left(\text { Afiliados }_{i t}\right)+u_{i t}
$$

$$
C M e_{i t}=X^{\prime}{ }_{i t} \beta+\gamma \ln \left(\text { Afiliados }_{i t}\right)+u_{i t}
$$

$C M e_{i t}=X_{i t}^{\prime} \beta+\gamma \ln \left(\right.$ Afiliados $\left._{i t}\right)+\eta D+u_{i t}$

Para los modelos semiparamétricos se planteó un modelo de regresión parcialmente lineal:

$C M e_{i t}=X^{\prime}{ }_{i t} \beta+g\left(\right.$ Afiliados $\left._{i t}\right)+\eta D+u_{i t}$

donde el vector $X$ reúne a las variables paramétricas y Afiliados es la variable no paramétrica. También se incluye el vector $D$, conteniendo las dummies que reflejan aquellos cambios regulatorios aplicados sobre el sector y discutidos en la sección previa. Dado que la estimación semiparamétrica del modelo parcialmente lineal requiere de la estimación no paramétrica entre la variable dependiente y la componente no paramétrica, en esta sección se presentan también los resultados de este ajuste. La estimación no paramétrica se obtuvo a través de técnicas de suavizado aplicadas localmente a los pares de observaciones (costos medios, afiliados). Esta estimación no asume una forma funcional entre ambas variables ni una distribución en particular para el término de error. Dado que las estimaciones no paramétricas no producen estimaciones de parámetros, los resultados se analizan en forma gráfica. 
En cada caso, los costos medios se explican por el número de afiliados como variable principal, para capturar el efecto de escala, que es el principal objeto de estudio de este análisis. La variable Afiliados es esperable que explique en forma significativa y negativa a los costos medios; ésto es, a mayor numero de afiliados, menor costo medio, estableciendo el efecto escala.

Para captar la relevancia de las economías de alcance, se incluye como variable el valor del fondo administrado por cada AFP: se espera que, a medida que los sistemas cuenten con mayores fondos, se incrementen las posibilidades de reducción de costos, para cualquier cantidad de afiliados. Sin embargo, como los fondos administrados se encuentran altamente correlacionados con la variable de afiliación, ello impide una estimación paramétrica de las economías de alcance. A partir de ello se decidió estimar este efecto solamente bajo la metodología semiparamétrica. El objetivo de incluir esta variable es permitir diferenciar entre los efectos de escala y de alcance de la cobertura de los sistemas de AFPs.

Completado el análisis de las estimaciones tanto paramétricas como semiparamétricas, se presenta entonces el Criterio de Información Bayesiano (BIC, por sus siglas en inglés) que permite seleccionar, entre los modelos estimados, al que mejor ajusta la relación entre costos medios por afiliados y el conjunto de variables analizadas, y para ambas metodologías aplicadas, paramétrica y semiparamétrica.

Por último, los gráficos permiten comparar, en primer lugar, ambos modelos de regresión simple, el paramétrico y el semiparamétrico, y en segundo lugar, los modelos completos o multivariados, incluyendo todas las variables explicativas seleccionadas (variables de conducta, de regulación, fusión, etc.) nuevamente para las estimaciones paramétricas y semiparamétricas. Para las estimaciones paramétricas de ambas metodologías se prefirió el ajuste mediante efectos fijos.

\subsection{Estimaciones}

El cuadro siguiente muestra para el caso de Bolivia seis regresiones. Las primeras tres corresponden a los ejercicios con modalidad paramétrica y las siguientes tres están vinculadas a la modalidad semiparamétrica. Asimismo, se presentan a continuación dos gráficos. El primero exhibe los ajustes de las regresiones simples para ambos métodos, paramétrico y no paramétrico, en tanto el segundo muestra el ajuste para las regresiones múltiples, para 
los métodos paramétrico y semiparamétrico (que incorpora en forma no paramétrica a la variable Afiliados).

Las regresiones paramétricas muestran que el logaritmo natural de afiliados presenta una relación negativa y significativa al 99\% al explicar los costos medios del sistema privado de pensiones del país, avalando la hipótesis de economías de escala.

La variable $w r$, que representa el costo de oportunidad del capital, muestra no ser significativa para cualquier especificación del modelo, en tanto que la variable $f \_r e n d$, que da cuenta de los rendimientos de los activos en los sistemas de pensiones privadas, establece una relación negativa y significativa en tres de las cinco regresiones. Ello refleja que el incremento de los rendimientos de las AFPs reduce el costo adicional de incorporar afiliados, en la medida en que se volverían más atractivas. Los traspasos por una parte, reflejados en la variable mer trasp, resultan significativos al 10\% sólo en la regresión paramétrica que excluye a las variables de regulación. Finalmente, la variable ff_valor, valor de los fondos administrados, no resultó relevante.

De las dos variables de regulación incorporadas, sólo $\mathrm{m} 1$ resultó ser significativa y positiva al 99\%. Esta variable indica la liberación de la cantidad de traspasos anuales por afiliado al sistema. Su signo establece claramente la presencia de un costo adicional surgido de la inclusión que esta normativa impuso al sistema. 


\section{Cuadro 3}

Bolivia. Estimaciones paramétricas y semiparamétricas

\begin{tabular}{|c|c|c|c|c|c|c|}
\hline & \multicolumn{3}{|c|}{ Regresiones paramétricas } & \multicolumn{3}{|c|}{ Regresiones paramétricas } \\
\hline & (1) & (2) & (3) & (4) & (5) & (6) \\
\hline In_af & $\begin{array}{l}-31.10 * * * \\
(-28.28)\end{array}$ & $\begin{array}{l}-33.40 * * * \\
(-16.61)\end{array}$ & $\begin{array}{l}-40.30 * * * \\
(-18.52)\end{array}$ & & & \\
\hline wr & & $\begin{array}{l}0.240 \\
(0.92)\end{array}$ & $\begin{array}{l}0.262 \\
(1.19)\end{array}$ & $\begin{array}{l}0.452 \\
(1.35)\end{array}$ & $\begin{array}{l}0.449 \\
(1.31)\end{array}$ & $\begin{array}{l}0.447 \\
(1.29)\end{array}$ \\
\hline f_rend & & $\begin{array}{l}-0.611^{* * *} \\
(-3.57)\end{array}$ & $\begin{array}{l}-0.496 * * \\
(-3.11)\end{array}$ & $\begin{array}{l}-0.380 * \\
(-2.26)\end{array}$ & $\begin{array}{l}-0.317 \\
(-1.19)\end{array}$ & $\begin{array}{l}-0.314 \\
(-1.16)\end{array}$ \\
\hline mer_trasp & & $\begin{array}{l}0.00381^{*} \\
(2.65)\end{array}$ & & $\begin{array}{l}0.00117 \\
(0.72)\end{array}$ & $\begin{array}{l}0.00136 \\
(0.81)\end{array}$ & $\begin{array}{l}0.00139 \\
(0.80)\end{array}$ \\
\hline $\mathrm{m} 1$ & & & $\begin{array}{l}5.510 * * * \\
(5.70)\end{array}$ & & $\begin{array}{l}-0.551 \\
(-0.35)\end{array}$ & $\begin{array}{l}-0.550 \\
(-0.34)\end{array}$ \\
\hline s1 & & & $\begin{array}{l}2.024 \\
(1.92)\end{array}$ & & $\begin{array}{l}-0.120 \\
(-0.09)\end{array}$ & $\begin{array}{l}-0.168 \\
(-0.11)\end{array}$ \\
\hline ff_valor & & & & & & $\begin{array}{l}-1.77 e-09 \\
(-0.08)\end{array}$ \\
\hline _cons & $\begin{array}{l}214.9 * * * \\
(33.80)\end{array}$ & $\begin{array}{l}232.6^{* * *} \\
(18.23)\end{array}$ & $\begin{array}{l}268.5^{* * *} \\
(20.59)\end{array}$ & $\begin{array}{l}-0.418 \\
(-1.06)\end{array}$ & $\begin{array}{l}-0.116 \\
(-0.17)\end{array}$ & $\begin{array}{l}-0.103 \\
(-0.15)\end{array}$ \\
\hline $\mathbf{N}$ & 78 & 70 & 70 & 70 & 70 & 70 \\
\hline $\mathrm{BIC}$ & 2,7406 & 2,9112 & 3,3991 & 2,1368 & 12,0168 & 36,7043 \\
\hline
\end{tabular}

Fuente: Elaboración propia en base a datos de la SPVS

Originalmente se propuso incorporar la variable de tendencia en la estimación. Sin embargo, y como se muestra en el gráfico 6, la correlación entre esta variable y el número de traspasos varía en distintos estadios temporales. Desde el inicio del sistema y hasta el año 2002, el número de traspasos no reviste significatividad, en tanto las firmas se encuentran en busca de nuevos afiliados en un mercado en crecimiento, lo que minimiza la competencia entre firmas. A partir del año 2002 se observa un aumento marcado en el número de traspasos vinculado con la variable $m 1$ incorporada al análisis. Este argumento refuerza la idea de retirar la variable tiempo de la estimación. 
Gráfico 6: Bolivia. Estimaciones paramétricas y semiparamétrica (a) Paramétrico y no paramétrico. Modelos simples

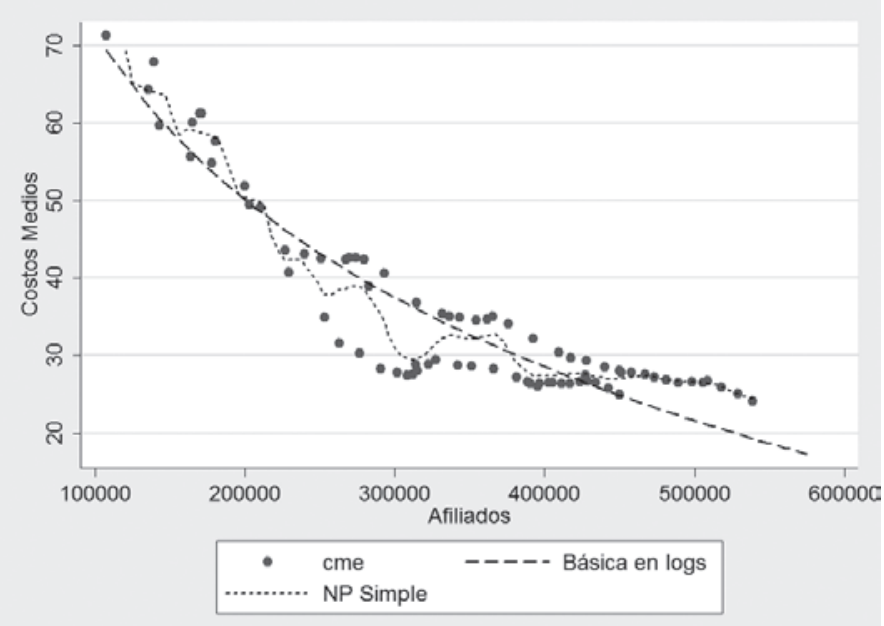

Referencias: Costos Medios (CM)

Fuente: Elaboración propia en base a datos de la SPVS.

(b) Paramétrico y semiparamétrico. Modelos completos

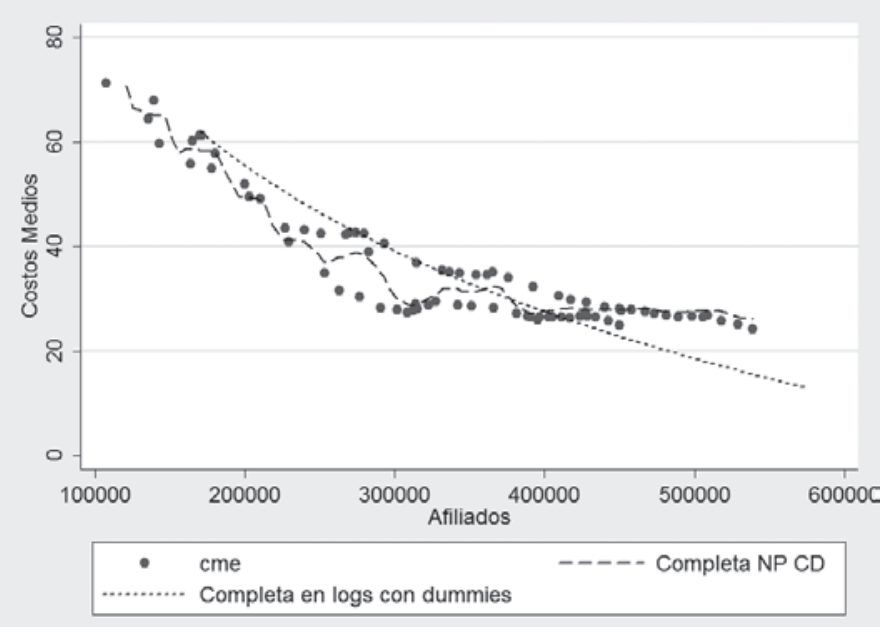

Fuente: Elaboración propia en base a datos de la SPVS Referencias: No Paramétrica con Dummies (NP CD) 
Finalmente, el ejercicio propone analizar la relación existente entre dos variables simultáneas: el crecimiento del número de afiliados en el tiempo, que hipotéticamente reduciría el costo medio por afiliados, y el incremento en el número de traspasos, de efecto inverso. El primero puede identificarse como un efecto cantidad, en tanto el segundo refiere a un efecto sustitución. El análisis de los coeficientes muestra que el efecto cantidad supera en intensidad y significancia al segundo. Esto se observa claramente en el gráfico 6 (b), donde la pendiente de la relación entre costos medios del sistema y afiliados se anula en el entorno de los 300 mil aportantes. De este modo, favorecer la competencia a partir de la regulación que facilita los traspasos se vuelve relevante en esta segunda etapa del desarrollo del sistema, en tanto permitiría seguir reduciendo sus costos medios.

Gráfico 7: Bolivia. Evolución de traspasos

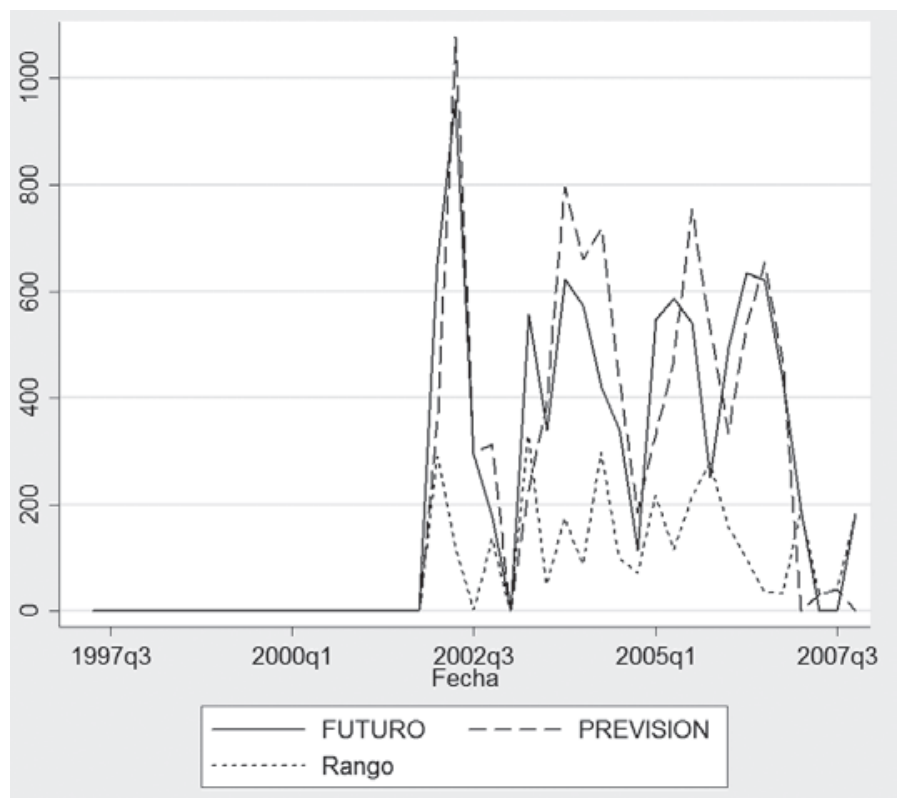

Fuente: Elaboración propia en base a datos de la SPVS

Por último, puede observarse que, según el criterio BIC en la última línea del cuadro 3, el modelo que mejor ajusta la relación entre los costos medios del sistema y el conjunto de variables propuestas es el modelo 4, que estima en forma semiparamétrica la relación entre los 
costos medios y los afiliados, controlando por los efectos del costo del capital los rendimientos de los fondos y el número de traspasos.

\section{Análisis de sistemas de comisiones}

El objetivo complementario de esta sección es presentar un marco analítico sobre los mecanismos a partir de los cuales se fija el cobro de las comisiones en los sistemas de pensiones, analizando las ventajas y desventajas de cada uno de ellos, y sus implicancias desde las miradas alternativas de los actores participantes en el mercado. Posteriormente se incluye una descripción de los mecanismos efectivamente utilizados en los sistemas bajo estudio.

En primer lugar, los mecanismos tradicionales de clasificación de comisiones reúnen tres opciones básicas:

- Sobre aportes: la AFP establece la comisión sobre una proporción definida del monto imponible del afiliado.

- Sobre la rentabilidad: el factor de cálculo de las comisiones se establece sobre la rentabilidad lograda por la AFP en la administración de los fondos.

- Sobre el saldo: la comisión de la firma se define sobre el monto acumulado por el afiliado en su cuenta de capitalización.

Cuál de estos esquemas de comisión resulta más conveniente a las AFPs dependerá de los beneficios asociados con la aplicación de cada uno de ellos, donde cada mecanismo de cobro se encuentra vinculado con un factor de riesgo propio, a partir del cual las AFP establecen sus beneficios probables.

Esquemáticamente, la estructura de costos de las AFPs puede ser dividida en dos componentes principales: uno primordialmente sistemático, vinculado con los gastos administrativos del sistema, y un segundo rubro, de Gastos de Gestión de Activos Financieros.

Los gastos administrativos se relacionan con el total de costos en que incurren las AFPs para la atención general de sus afiliados, servicios de administración y mantenimiento de cuenta (backoffice), seguros y otras erogaciones vinculadas; en tanto los Gastos de Gestión de Activos se vinculan a la administración de los fondos y por ello tienen un mayor grado de variabilidad asociado con su riesgo financiero. El primero está directamente identificado con 
la eficiencia en la gestión organizacional, y se traduce en la estructura de costos, mientras que el segundo tiene su correlato con la eficiencia en la gestión de la cartera financiera.

Cada tipo de comisión distribuye en forma diferente entre afiliados y AFPs la carga de riesgo de cada uno de estos componentes de gasto. Con una comisión sobre aportes, la carga recae exclusivamente sobre el afiliado: sin importar cuáles sean los resultados financieros de las AFPs, éstas obtienen el mismo monto en concepto de comisiones.

Las comisiones sobre rentabilidad, por el contrario, hacen que el total del riesgo recaiga sobre las AFPs pues ata sus ingresos a la administración de los fondos.

Por último, las comisiones sobre saldo distribuyen la carga del riesgo, dado que el saldo de la cuenta depende tanto del ingreso del afiliado como de la rentabilidad obtenida sobre esa cuenta.

A fin de comparar el efecto de cada mecanismo de imposición para la definición de las comisiones, se requiere analizar en cada caso la reacción estratégica de los actores participantes (afiliados, AFPs y entes reguladores) ante cada uno de ellos.

Para ello es necesario considerar que las AFPs tienen como función-objetivo maximizar sus beneficios, en tanto que los afiliados persiguen alcanzar un máximo de sus ingresos jubilatorios netos. Por su parte, el ente regulador requiere, en representación del conjunto de la población afiliada al sistema, maximizar el bienestar agregado. Sin embargo, y dado que el mercado de pensiones privadas se desenvuelve dentro de un marco de incertidumbre y de información asimétrica entre actores, estas premisas no se cumplen con certeza: no se conocen los rendimientos de los activos futuros, y sólo es posible definir tablas de vida para promedios de grupos sociales. Por tanto, los mecanismos de ingresos y rendimientos no son conocidos por los actores. Asimismo, los afiliados, como especialmente las AFPs, cuentan con información privada relevante para la toma de decisiones.

En el caso de los beneficiarios, existe información imperfecta sobre las preferencias de cada uno de ellos, tanto en términos de expectativas de contribución presente como de sus preferencias intertemporales de ingreso. En el caso de las firmas participantes del mercado, fundamentalmente, cada una de ellas cuenta con información privada sobre sus estructuras de costos, las cuales sólo pueden ser inducidas a partir de las señales enviadas (esquema de señalamiento o signalling) y mediante la estimación de funciones de costos mediante paneles de datos. En todo caso, las mismas AFPs, mediante el accionar competitivo del mercado, 
como los afiliados y los mismos entes reguladores, resignan ganancias sociales o privadas al operar en un esquema de información incompleta e imperfecta.

A partir de ello, las estructuras de comisiones y sus modos de fijarlas construyen sobre tal falla de mercado, estableciendo mecanismos de absorción y transferencia de riesgos entre actores. El ente regulador, dentro de ese contexto, arbitra sus instrumentos de intervención, procurando alinear los intereses de los distintos participantes, en procura del mayor interés social.

Ante un esquema de comisiones sobre aportes, éste puede tener dos tipos de componentes: uno fijo y uno variable. El componente fijo es aquél que no varía a través de los ingresos de los distintos tipos de afiliados, mientras que el componente variable sí lo hace. En caso de que el marco regulatorio diera libertad sobre la elección en el peso de cada componente, las AFPs tenderían a ofrecer un mix entre ambos, asociado con los distintos tipos de afiliados.

Estos afiliados, por su parte, buscando minimizar sus costos, buscarían mantener un componente variable reducido y un componente fijo mayor, a medida que se incrementan sus ingresos. Como consecuencia, las AFPs maximizarían beneficios al ofrecer distintos paquetes para cada uno de los tipos de afiliados. Sin embargo, y en la medida que las AFPs se encuentran más informadas sobre la administración de fondos que sus afiliados, la asimetría limita la elección óptima de tal grupo, brecha que se amplía en el caso de los afiliados de bajos ingresos y menor información disponible.

De tal modo, es muy probable que se alcance un esquema donde el componente fijo no varíe demasiado a través de los ingresos de los afiliados y exista una carga mucho mayor sobre los sectores de menores ingresos. A su vez, en la búsqueda de maximización de beneficios, las AFPs pueden decidir no afiliar a grupos de bajos ingresos. Así, los resultados de bienestar bajo un esquema de comisiones sobre aportes con componentes fijos y variables serán perjudiciales para el bienestar agregado, especialmente para los sectores de bajos ingresos, debido a la presencia de selección de riesgo.

Bajo un esquema de comisiones variables, subsisten diversos elementos de interés para el regulador. En primer lugar, este esquema, como se mencionó anteriormente, carga la totalidad del riesgo financiero de la AFP sobre los afiliados. 
En segundo lugar, con libertad de elección de la tasa variable, las AFPs pueden buscar incrementar este porcentaje, a medida que se incrementan los ingresos de sus afiliados, haciendo progresiva su tasa de ganancia.

En tercer lugar, debido a la información asimétrica existente tanto entre las AFPs y los afiliados como entre los mismos afiliados, el esquema puede resultar en una sucesión de comisiones, con porcentajes muy altos para los de bajos ingresos y menos informados, y muy bajas para los de mayores ingresos, arribando a un esquema tarifario regresivo (Varian, 1981).

En cuarto lugar, los sectores de bajos ingresos, dada la relativa baja rentabilidad generada para la firma, pueden resultar excluidos del mercado. Para todos estos casos, una solución, encontrada en la mayor parte de los países de la región latinoamericana que utilizan estos esquemas de jubilación, es la fijación de una misma tasa para todos los afiliados de una misma AFP, aunque con capacidad de variar entre las AFPs. De este modo, el mecanismo de competencia entre AFPs mediante porcentajes fijos de comisiones tiende a compensar el efecto potencial de discriminación por ingreso.

Por su parte, un esquema de comisiones sobre rentabilidad busca incentivar a las AFPs a alcanzar la cartera más rentable del mercado, bajo el supuesto de que, a más rentabilidad obtenida, mayores serán los beneficios generados a la firma. Este esquema implica, de cualquier modo, una absorción de riesgo por parte de cada empresa, que encontrará un mecanismo adicional de diferenciación en el mercado a partir de los rendimientos obtenidos. Ello, por su parte, la enfrentará a la necesidad de establecer un mecanismo de definición de cartera que se adecuará a la naturaleza de toma de riesgo relativa de cada AFP.

La característica de este tipo de comisión es que el total de la carga de riesgo de mercado recaiga sobre las AFPs, protegiendo en principio a los afiliados del sistema. Aun así, pueden existir consecuencias estratégicas a considerar en cada mercado. En la medida en que ciertas AFPs no necesariamente estén dispuestas a absorber el riesgo del sistema, o que por su escala no se encuentran en condiciones de brindar mejores rendimientos, dejarán el mercado. Ello, conjuntamente con la naturaleza del sector caracterizado por un amplio rango de economías de escala, mostradas en la primer parte de éste trabajo, puede llevar a mercados altamente concentrados.

Por último, se identifica el esquema de cálculo de las comisiones sobre el saldo de la cuenta acumulado del afiliado. Este tipo de comisión amplía la visión de las AFPs para la 
obtención de sus ingresos, en la medida que deban considerar todo el flujo de la cuenta del afiliado, donde están interconectados sus ingresos junto con la rentabilidad alcanzada por la AFP por la administración de esa cuenta.

Este tipo de comisiones haría que la AFP maximice una función de beneficios que pondere el ingreso presente y el futuro: cobrar comisiones demasiado altas al comienzo implica menores fondos para capitalizar a futuro, lo que incentiva una elección estratégica sobre la corriente de imposición sobre los aportes a lo largo del tiempo de contribución del afiliado. En cierta medida, este modelo de cobro combina los dos esquemas previos, en tanto la comisión sobre saldo es la resultante de una suma ponderada de los otros dos modelos.

Este modelo, por tanto, genera para la AFP un incentivo para incrementar el porcentaje de aportes a los afiliados cercanos a la fecha de retiro. Por otro lado, y al igual que surge del análisis de los dos componentes anteriores por separado, requiere de un marco regulatorio que limite la discriminación hacia los sectores de menores ingresos. La regulación en este caso debe abarcar dos aspectos: las comisiones según el ingreso entre afiliados en un mismo momento del tiempo y la variación de esa comisión a lo largo del tiempo para el mismo afiliado. Una alternativa puede vincularse con la fijación de una comisión sobre saldos para los individuos con ingresos iguales o menores a un determinado umbral, y la fijación de otro nivel de comisiones para los individuos que se encuentran cerca de la fecha de retiro. De otro modo, puede considerarse el establecimiento de comisiones según el saldo acumulado por el afiliado, previa aprobación del mismo por la autoridad regulatoria y con la libertad de los afiliados de elegir el prospecto que les resulte más atractivo en caso de existir varios de éstos.

En el caso de Bolivia, las Administradoras de Fondos de Pensiones han actuado durante el período analizado en base a los siguientes tipos de comisiones:

- una comisión por el servicio de afiliación, procesamiento de datos y administración de prestaciones fijada en $0.5 \%$ del total ganado o del ingreso cotizable del afiliado,una comisión por el servicio de recaudación y acreditación de las primas de los seguros,

- una comisión competitiva por servicio de pago de pensiones del Seguro Social Obligatorio de largo plazo, establecida en un tope máximo de $1.31 \%$ del monto de la pensión que corresponda pagar (aprobada mediante RA-SPVS-IP- No.963/2002 de 11/ $\mathrm{Dic} / 2002), \mathrm{y}$ 
- una comisión por el servicio de administración del portafolio, en base a la Resolución Administrativa SPVS-IP-No124 de 29 de junio de 1999. Se contabiliza diariamente sobre el valor del fondo de acuerdo a los siguientes tramos:

\begin{tabular}{|l|l|}
\hline $\begin{array}{l}\text { Para montos del portafolio inferiores } \\
\text { o iguales a u\$s. } 1.000 \text { millones. }\end{array}$ & $0.2285 \%$ de esa porción del portafolio. \\
\hline $\begin{array}{l}\text { Para montos del portafolio mayores } \\
\text { a u\$s. } 1.000 \text { millones, pero inferiores } \\
\text { o iguales a u\$s. } 1.200 \text { millones. }\end{array}$ & $0.0140 \%$ de esa porción del portafolio. \\
\hline $\begin{array}{l}\text { Para montos del portafolio mayores } \\
\text { a u\$s. 1.200 millones, pero inferiores } \\
\text { o iguales a u\$s. } 1.500 \text { millones. }\end{array}$ & $0.0067 \%$ de esa porción del portafolio. \\
\hline $\begin{array}{l}\text { Para montos del portafolio } \\
\text { superiores a u\$s. 1.500 millones }\end{array}$ & $0.0000 \%$ de esa porción del portafolio. \\
\hline
\end{tabular}

A pesar de que la comisión por administración del portafolio es anual, se devenga diariamente sobre el valor de los fondos. Por otra parte, la comisión de administración de portafolio se cobra tomando en cuenta la suma del Fondo de Capitalización Individual (las cuentas individuales, el seguro de riesgo común y el profesional/laboral) y el Fondo de Capitalización Colectiva (compuesto por las acciones de las empresas capitalizadas y la liquidez generada por estos activos). A diciembre de 2006, las AFPs han cobrado la comisión de administración de portafolio de acuerdo a lo establecido en la resolución administrativa antes citada. Al 31 de diciembre de 2006 y según lo establecido en la Ley del Bonosol, que determina que la comisión por administración del portafolio será establecida en función del valor y rendimiento, no se ha aplicado dicha modificación normativa, ni se ha realizado una adecuación a los contratos.

Por otra parte, se pagan con cargo al fondo que administran las AFPs los siguientes gastos: (a) Comisión a la entidad de depósito de valores, por mantenimiento y anotaciones en cuenta de los valores custodiados; $0.023 \%$ para valores de renta fija y $0.076 \%$ para valores de renta variable, sobre valor nominal del Fondo, y (b) otros gastos de transacción.

\section{Conclusiones}

Este trabajo propone un aporte al análisis de los sistemas de pensiones privadas en Bolivia, en la medida que permite corroborar y completar, mediante criterios adicionales, los hallazgos realizados por la literatura sobre el particular hasta la fecha. Por su parte, el uso de 
mecanismos paramétricos y semiparamétricos aporta al enriquecimiento de las técnicas de implementación utilizadas hasta el momento.

El eje central de este proyecto de investigación (que abarcó el estudio de otros siete casos nacionales) se ha centrado en el análisis de los costos medios de los sistemas de pensiones privadas, a fin de identificar la presencia de economías de escala y de alcance en estos mercados.

La propuesta incorporó asimismo la posibilidad de testear otras hipótesis de trabajo vinculadas con los determinantes de los costos medios. Entre ellos se encuentra la identificación de la importancia de las estrategias privadas desplegadas sobre la estructura de costos de los diferentes sistemas, tales como diferencias en mecanismos de captura de afiliados mediante gastos de comercialización, como también el peso de las acciones públicas, mediante innovaciones regulatorias y su impacto en la evolución de los costos del sistema.

A pesar de las marcadas diferencias existentes entre los sistemas nacionales de pensiones privadas, definidos en términos de cantidad de afiliados, número de empresas participantes y peso específico del sistema sobre el total de población económicamente activa, algunas conclusiones resultan similares. Ellas se refieren a cierta homogeneidad en las tasas de rendimiento y su relativamente pobre impacto sobre los costos medios del sistema, y una relación significativa y negativa entre las dos variables principales del estudio (costos medios y número de afiliados). Ello refuerza las hipótesis tradicionales de los mercados de servicios, y en particular contribuye a la robustez de los hallazgos alcanzados en la literatura previa.

Asimismo, el trabajo propone un debate metodológico sobre la relación entre la estructura de las comisiones y el efecto de la norma regulatoria sobre los costos medios y el número de afiliados al sistema.

Como fuera presentado en la sección anterior, la vinculación entre la estructura de comisiones de un sistema y las funciones de costos de las firmas participantes se encuentra intermediada a partir del efecto de las primeras sobre el comportamiento estratégico de los actores que influye inicialmente sobre la demanda del mercado. Subsecuentemente, la respuesta de los afiliados refleja determinados patrones de elección de AFPs que localizan a los sistemas en general y a las firmas en particular en ciertos tramos de la función de costos. Como consecuencia, se identifican economías de escala y rentas de eficiencia en la concentración del sistema, lo que requiere particularmente de un papel atento de las instituciones regulatorias. 
Del mismo modo, el análisis econométrico encuentra que el efecto de las normas regulatorias en gran parte de los casos no parecen estar operando directamente sobre los costos medios, sino a través de la variable vinculada con el número de afiliados. Ello resulta respaldado tanto por la pérdida de ajuste de las regresiones que contienen dummies regulatorias por sobre aquéllas que no las tienen, como en las correlaciones comparadas de tales dummies sobre las variables de costos y de afiliados.

En el primer caso, y como se observa en los cuados referidos a las implementaciones econométricas del país, las regresiones paramétricas y particularmente semiparamétricas que contemplan variables independientes regulatorias alcanzan valores relativamente más altos en los criterios de comparación utilizados.

Finalmente, el trabajo muestra ser un instrumento idóneo para responder algunas de las preguntas planteadas en el plan de trabajo original, como también es la fuente de nuevas inquietudes, especialmente en la investigación de cambios regulatorios particulares, como la liberación de traspasos y la estimación de Escalas Mínimas Eficientes. La posibilidad de actualizar la información al presente permitiría identificar la situación presente con respecto al período analizado y los cambios ocurridos durante el último lustro.

Fecha de recepción: 30 de mayo de 2013 Fecha de aceptación: 15 de abril de 2014 


\section{Referencias}

1. Asociación Internacional de Organismo de Supervisión de Fondos de Pensiones, AIOS (2007). "La capitalización individual en los sistemas de pensión en Latinoamérica". Disponible en: http://www.aiosfp.org

2. Aguilera, N. (2004). "A Note on International Administrative Charges of Funded Pensions". Journal of Social Security, 251, 8-13.

3. Aguilera,N.yVelásquez,C.(2005)."Economíasdeescalaenlaindustriadelasadministradoras de fondos de pensiones, un enfoque semiparamétrico". Premio de Pensiones. 2005. Disponible en: http://www.consar.gob.mx/convocatoria_2007/index.html Consulta: 15 de mayo de 2007

4. Apella, I. y Maceira, D. (2004) Economías de escala y barreras a la entrada en el mercado argentino de AFPJ. Argentina: CEDES. Disponible en: http://www.aaep.org.ar/espa/anales/works06/Apella_Maceira.pdf

5. -------- (2005). “Economías de la regulación: una perspectiva teórica”. Mimeo, UBASAFJP.

6. -------- (2006). "Alternativas ante la separación de funciones en el mercado argentino de AFJP”. Mimeo, UBA-SAFJP.

7. Auguste, S. y Urbiztondo, S. (2004). "La reforma previsional en Argentina y en América Latina: un diagnóstico alternativo de sus aspectos más críticos”. Documento de trabajo. Fundación de Investigaciones Económicas Latinoamericanas, FIEL.

8. ------- (2008). Sistemas privados de capitalización en América Latina: un estudio comparado, Instrumentos Regulatorios. Banco Interamericano de Desarrollo.

9. Berdejo, M.A., Galarza, B. y Nagamine, J. (2006). "Mecanismos para incentivar la competencia en los sistemas de pensiones basados en capitalización individual". Documento de trabajo 01/2006, Superintendencia de Banca, Seguros y AFP, Perú.

10. Box, G. y Cox, D., (1964). "An Analysis of Transformation”, Journal of the Royal Statistical Society. Series B (Methodological), 26 (2), 211-252.

11. CEF (2008). Sistemas privados de capitalización en América Latina: un estudio comparado Estructura de Mercado, Costos y Comportamiento Estratégico de la Oferta. Argentina, Chile, México y Perú. Banco Interamericano de Desarrollo 
12. Chamberlin, E. (1956). The Theory of Monopolistic Competition. Seventh Edition, Cambridge University Press.

13. Chevalier, Judith y Ellison, Glenn (1997). "Risk Taking by Mutual Funds as a Response to Incentives”. Journal of Political Economy, University of Chicago Press, 105(6),11671200, December.

14. Chisari, O., Valdés-Prieto, S., Quesada, L., Dal Bo, P. y Rossi, M., (1998). "Opciones estratégicas en la regulación de las AFJP: costos, comisiones y organización de la industria del régimen de capitalización". Superintendencia de Administradoras de Fondos de Jubilaciones y Pensiones.

15. Epanechnikov, V., (1969). "Nonparametrics Estimates of a Multivariate Probability Density”. Theory of Probability and its Applications, 14, 153-158.

16. Galarza, B. y Olivera, J., (2001). "La industria en el sistema privado de pensiones de Perú". Documento de trabajo 02/2001, Superintendencia de Banca, seguros y AFP, Perú.

17. García, M. y Rodríguez, T. (2003). "La organización del mercado de ahorro para el retiro mexicano durante su etapa de acumulación”. Tesis ITAM, 2003.

18. Hsiao, C., Li, Q. y Racine, J. S. (2007). "A consistent model specification test with mated categorical and continuous data”. Journal of Econometrics 140 (2), 802-826.

19. Li, Q. y Racine, J. S. (2004). Nonparametric Econometrics: Theory and Practice. Princeton University Press.

20. Masías, L. y Sánchez E. (2007). "Competencia y reducción de comisiones en el sistema privado de pensiones: el caso peruano”. SBS Documento de trabajo No2. Superintendencia de Banca, Seguros y Administradoras Privadas de Fondos de Pensiones.

21. Meléndez, J., (2004). "La industria de la Afore: un análisis de su estructura y recomendaciones de política de competencia y regulación”. Mimeo, IMSS.

22. Pagan, A. y Ullah, A. (1999). Nonparametric Econometrics. New York: Cambridge University Press.

23. Racine, J. S. (2008). "Nonparametric Econometrics: A Premier". Foundations and Trends in Econometrics, 3 (1).

24. Robinson, P.M. (1988). “Root-n Consistent Semiparametric Regression”. Econometrica, 56: 931-954. 
25. Zepeda, J.y Roldán, O. (2005). “Las Afores: empresas de escala reducida”. Documento de trabajo 2005-1, Comisión Nacional del Sistema de Ahorro para el Retiro.

\section{Fuentes de información de datos sobre regulaciones:}

- http://www.clad.org.ve/siare/basesju/bseguridad.html

- http://www.ilo.org/dyn/natlex/natlex_browse.details?p_lang=en\&p_ classification $=15.06 \& p \_$origin $=C O U N T R Y \& p \_s o r t b y=S O R T B Y \_C O U N T R Y \& p \_$ country=PER\&p_country_all_any=ALL\&p_keyword_all_any=ALL\&p_ start $=51 \& p \_$increment $=50$ 